Review

\title{
Polyamines under Abiotic Stress: Metabolic Crossroads and Hormonal Crosstalks in Plants
}

\author{
Marta Bitrián, Xavier Zarza, Teresa Altabella, Antonio F. Tiburcio and Rubén Alcázar * \\ Unit of Plant Physiology, Department of Natural Products and Plant Biology, Faculty of Pharmacy, \\ University of Barcelona, Diagonal, 643, 08028 Barcelona, Spain \\ * Author to whom correspondence should be addressed; E-Mail: ralcazar@ub.edu; \\ Tel: +34 934024492; Fax: +34 934029043.
}

Received: 22 June 2012; in revised form: 6 August 2012 / Accepted: 10 August 2012 /

Published: 20 August 2012

\begin{abstract}
Polyamines are essential compounds for cell survival and have key roles in plant stress protection. Current evidence points to the occurrence of intricate cross-talks between polyamines, stress hormones and other metabolic pathways required for their function. In this review we integrate the polyamine metabolic pathway in the context of its immediate metabolic network which is required to understand the multiple ways by which polyamines can maintain their homeostasis and participate in plant stress responses.
\end{abstract}

Keywords: polyamines; stress; metabolism; SAM; GABA; proline; ABA

\section{Introduction}

Abiotic stresses such as cold/freezing, salinity, heat and drought represent serious threats to agriculture. Climatic change is predicted to increase global temperature, alter precipitation patterns and intensify drought, increasing the need to grow crops in saline soil [1,2]. Plants, which are sessile organisms, have evolved metabolic and hormonal pathways to cope with environmental challenges. The study of this natural evolution on stress responsiveness is providing new leads to crop protection. In recent years, genetic and genomic approaches have revealed complex metabolic and hormonal networks which are coordinated to provide an optimal response to stress conditions. Efforts have created network models of stress and hormone regulatory pathways, as well as the definition of frameworks of co-regulated target genes of abiotic stress response pathways e.g. [3]. It is generally recognized that the study of stress responses must be integrated in the context of a multiple response 
involving different plant metabolites and hormones rather than in isolated pathways. Global transcriptional and metabolomic approaches very frequently recognize the participation of polyamines (PAs) in a large number of abiotic and biotic stresses. In this review we synthesize what is known about direct interactions of PAs with other metabolic pathways during the stress response. We also discuss the dependence of PA responses on the stress hormone abscisic acid (ABA) during drought.

\section{Polyamine Biosynthesis and Its Interaction with Other Metabolic Pathways}

PAs are polycationic compounds of low molecular weight which are present in most living organisms [4]. The diamine putrescine (Put), triamine spermidine (Spd), tetramines spermine (Spm) and thermospermine (tSpm) can be found in free (Figure 1) and conjugated forms. The homeostasis of PAs in the cell is mainly achieved through the regulation of its biosynthesis and catabolism. However, polyamine (PA) conjugation in form of hydroxycinnamic acid amides such as coumaroylputrescine, feruloylputrescine, dicoumaroylspermidine, diferuloylspermidine or diferuloylspermine among others, significantly contributes to the regulation of free PA levels in plants [5]. Through the regulation of PA biosynthesis, catabolism and conjugation, the free levels of PAs are tightly regulated and generally only oscillate in response to environmental insults (e.g. stress) or during the transition between different developmental stages (e.g. flowering). In the following sections we integrate the PA pathway in the context of a wider metabolic network and discuss current evidences supporting a role for PAs in drought protection.

Figure 1. Chemical structure of putrescine, spermidine, spermine and thermospermine. Aminopropyl moieties (in red) are added to the four carbon linear chain skeleton of putrescine (in blue) to produce spermidine, spermine and thermospermine.

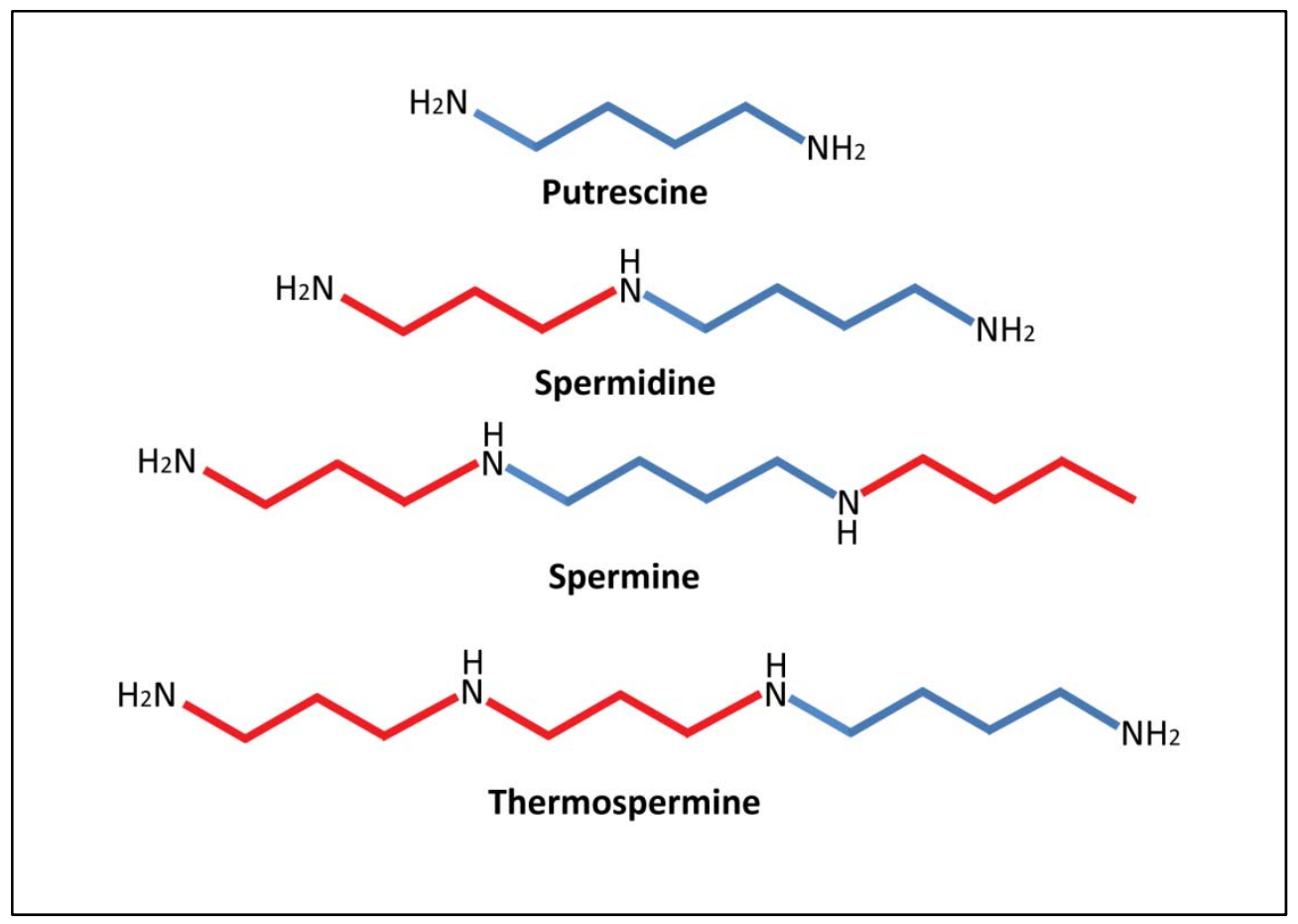




\subsection{Biosynthesis of Putrescine. Interactions with Proline, Urea, NO and Alkaloid Biosynthesis}

The first PA synthesized in the PA-bioynthetic pathway is Put (Figure 2). This PA can be derived from the decarboxylation of the amino acid ornithine through an enzymatic reaction catalyzed by ornithine decarboxylase (ODC, EC 4.1.1.17; Figure 2). Whereas this enzymatic step is generally recognized to be present in all living organisms, evidences are found that the model species Arabidopsis thaliana does not have a functional ODC pathway [6]. In addition, some of the regulatory mechanisms involved in the regulation of ODC activity in mammals, such as the ubiquitin-independent proteasome degradation of ODC by a PA-induced protein called 'antizyme', are not found in plants $[7,8]$. Hence, it is likely that the ODC pathway, as well as the regulation of ODC activity, has evolved differently in plants compared to mammals, leading to an eventual loss of this pathway in some plant species studied. Loss of ODC pathway might be due to the evolution of an alternative route to Put biosynthesis present in plants and bacteria [4], the arginine decarboxylase (ADC) pathway, which uses arginine as substrate for Put biosynthesis (Figure 2).

Figure 2. Biosynthesis of polyamines and connections with other metabolic pathways. ACC, aminocyclopropane carboxylic acid; ACL5, ACAULIS5 - thermospermine synthase; ADC, arginine decarboxlase; AIH, agmatine iminohydrolase; CPA, N-carbamoylputrescine amidohydrolase; dcSAM, decarboxylated SAM; $\delta$-OAT, $\delta$-ornithine aminotransferase; ODC, ornithine decarboxylase; P5CDH, pyrroline 5-carboxylate dehydrogenase; PMT, Putrescine N-methyltransferase; SAM, S-adenosylmethionine; SAMDC, S-adenosylmethionine decarboxylase; SPDS, spermidine synthase; SMPS, spermine synthase.

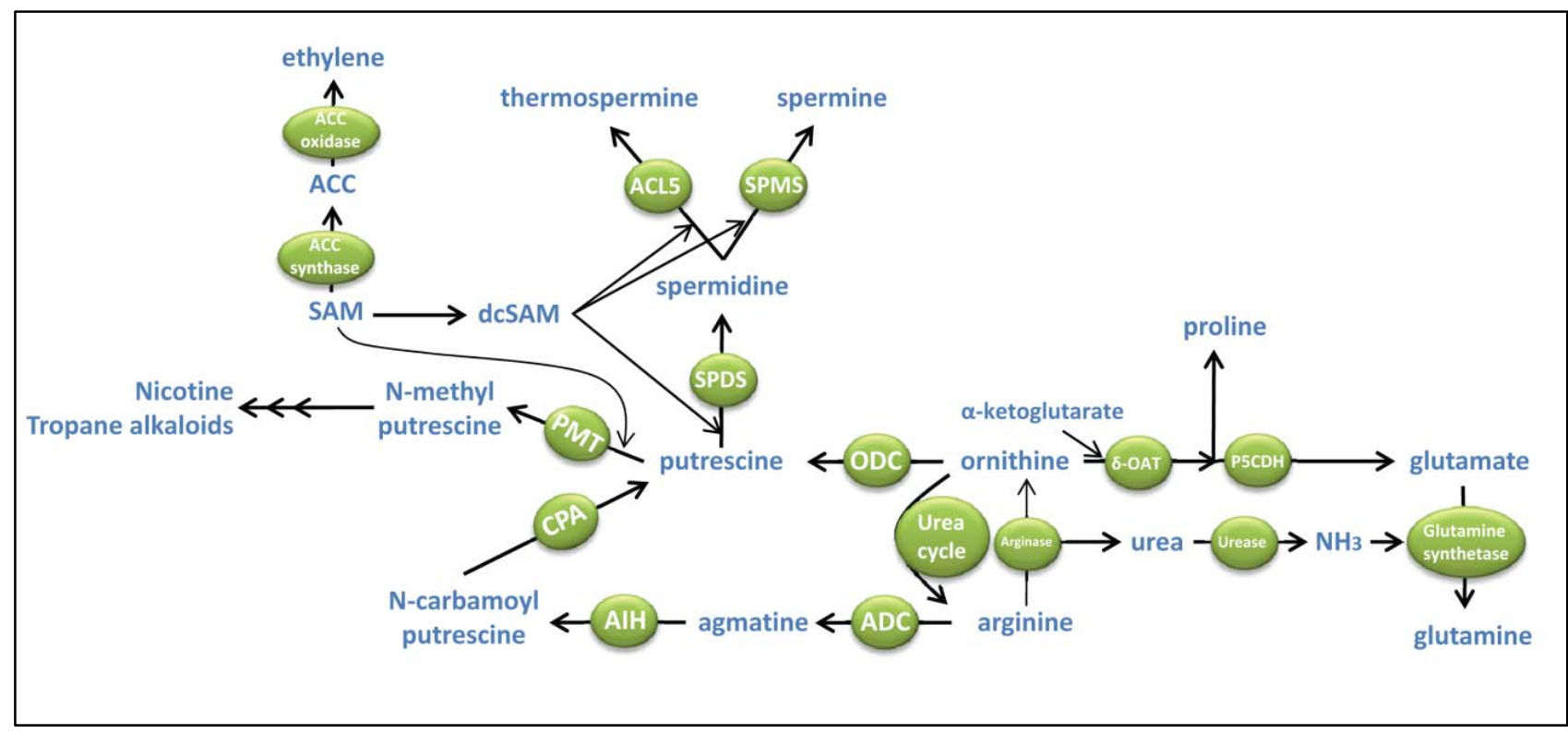

Arginine to put conversion requires three consecutive enzymatic reactions (Figure 2). The first step is the enzymatic decarboxylation of arginine by ADC (EC. 4.1.1.19). The product of this enzymatic reaction is agmatine, which serves as substrate for agmatine iminohydrolases (AIH, EC. 3.4.3.12) to produce N-carbamoyl-Put. The final step in the ADC pathway is the hydrolysis of N-carbamoyl-Put to Put by N-carbamoyl-Put amidohydrolases (CPA, EC 3.5.1.53). Despite the need of three consecutive 
enzymatic reactions for the arginine to Put conversion, evidences indicate that the limiting enzyme in Put biosynthesis is ADC [4,9]. Hence, manipulation of Put biosynthesis in plants can be achieved by the genetic manipulation of ADC activity such as over-expression of ADC encoding genes. In this way, plants accumulating high levels of endogenous Put have been generated and showed enhanced resistance to drought and freezing stresses [4,9,10]. Except for few cases [9], increases in Put levels achieved by overexpression of $A D C$ are moderate which suggests the occurrence of post-transcriptional regulation of $A D C$, transformation of Put to higher PAs (e.g. Spd, Spm and tSpm), catabolism/conjugation, among others mechanisms to maintain Put homeostasis.

Even though ADC and ODC use different substrates, both pathways for Put biosynthesis are connected by arginase, an enzyme that converts arginine to ornithine. Arabidopsis thaliana contains two genes coding for arginase (ARGAH1 and $A R G A H 2)$ which can complement the yeast arginase deficient (carl) mutant [11]. The expression of $A R G A H 1$ is restricted to pollen whereas $A R G A H 2$ expression is induced in leaves by treatment with methyl jasmonate [12]. Flores et al. [13] also reported $A R G A H 1$ and $A R G A H 2$ expression in expanded cotyledons and root vasculature of young seedlings, especially in response to the synthetic auxin NAA. ARGAH2 is a major contributor to arginase activity in seedlings and both ARGAH1 and ARGAH2 are localized to the mitochondrial matrix [13]. Interestingly, over-expression of ARGAH2 in Arabidopsis thaliana leads to enhanced resistance to Botrytis cinerea, thus suggesting a role for arginase in the biotic stress responses to necrotrophic pathogens at least in this species [14].

The hydrolisis of arginine to ornithine is also a source of urea [15] (Figure 2). The ornithine formed by arginase activity can be used for PA biosynthesis in plants provided with an ODC pathway. Alternatively, ornithine can be converted again into arginine through the urea cycle. Another metabolic pathway for ornithine is its catabolism through the activity of ornithine- $\delta$-aminotransferases $(\delta$-OAT, Figure 2). In this enzymatic reaction, the side chain amino group of ornithine is transferred to $\alpha$-ketoglutarate to generate glutamate and pyrroline 5-carboxylate. The latter compound can be oxidized to glutamate by pyrroline-5-carboxylate dehydrogenase $(\mathrm{P} 5 \mathrm{CDH})$ or alternatively, be reduced by NADPH to generate proline (Figure 2). However, it is still controversial the contribution of $\delta$-OAT to proline levels since oat mutants in Arabidopsis thaliana do not show altered proline levels under stress [16]. Therefore, it is more likely that the final product from $\delta$-OAT activity is glutamate rather than proline. The urea released by arginase activity is exported to the cytosol and hydrolized by cytosolic ureases to ammonia (Figure 2). Ammonia is then re-assimilated by cytosolic glutamine synthetases to glutamine using the glutamate derived from $\delta$-OAT catabolism (Figure 2).

Overexpression of ARGAH2 in Arabidopsis thaliana has been shown to lead to increased arginase activity and reduced arginine levels which did not result in lower PA levels [14]. This might be the consequence of the reallocation of free and conjugated PA forms, inhibition of PA catabolism, its transport or other mechanistic processes by which plants maintain PA levels under strict control. The requirement of a minimum PA pool for cell survival likely underlies its tight homeostasis regulation [4]. Interestingly, even though $\delta$-OAT activity does not necessarily lead to increased proline levels [16], overexpression of $A R G A H 2$ lead to increased proline in some lines [14]. Further genetic analyses would be required to better define the metabolic interaction between arginine and proline mediated by $\delta$-OAT. 
In animal cells, arginine is also susbtrate for nitric oxide synthases (NOS) which produce nitric oxide (NO). Nitric oxide plays important roles in the regulation of stomatal movements in response to ABA and is an integral part in many defense and developmental pathways. Although the identification of a NOS orthologue gene in plants that would link arginine to NO biosynthesis is missing $[17,18]$ some reports evidence that PAs, and particularly Spm, induce a rapid NO production without lag [19]. Increased NO observed in arginase mutants argah1-1 and argah2-1 has been suggested to derive from an increased availability of arginine for PA biosynthesis [13]. The source for NO production induced by PAs still remains to be elucidated [20].

In the crossroad of PAs with other metabolic pathways, the interaction of Put with secondary metabolism is well established in some plants. The enzyme Putrescine N-methyltransferase (PMT, Figure 2) catalyzes the methylation of Put using SAM as methyl donor. The product of this enzymatic reaction, N-methyl putrescine, is required for the synthesis of nicotine, tropane and nortropane alkaloids in Solanaceae and Convolvulaceae plants [21].

\subsection{Biosynthesis of Spermidine, Spermine and Thermospermine. Interactions with S-adenosylmethionine (SAM) and ethylene}

Higher molecular weight PAs spermidine and spermine are synthesized by the sequential addition of aminopropyl moieties to the four carbon linear chain skeleton of Put (Figure 1) through enzymatic reactions catalyzed by Spd and Spm synthases, respectively (SPDS, EC 2.5.1.16 and SPMS, EC 2.5.1.22; Figure 2).

The donor of aminopropyl groups is decarboxylated S-adenosyl methionine (dcSAM) which is synthesized from decarboxylation of S-adenosyl methionine (SAM) by SAM decarboxylases (SAMDC, EC 4.1.1.50; Figure 2). The availability of dcSAM limits the biosynthesis of Spd and Spm [22]. This makes SAMDC one of the major regulators of the PA biosynthetic pathway. Furthermore, SAMDC competes for SAM, which is used as universal methyl donor in many enzymatic reactions involving $\mathrm{O}-, \mathrm{N}$ - and $\mathrm{C}$-methyltransferases in primary and secondary metabolism [23]. dcSAM can only be used for PA biosynthesis and therefore, the regulation of SAMDC activity is likely to be relevant in the context of a complex metabolic network. Indeed, the transcriptional regulation and biochemical properties of SAMDCs are tightly regulated and have been extensively studied [24]. As anticipated, SAM is precursor of other metabolites in plants. Relevant for crop stress protection against nutritional deficiency is the requirement of SAM for the synthesis of nicotianamine by nicotianamine synthase [25]. Nicotianamine is a strong chelator of iron and in graminaceous plants is precursor of phytosiderophores required for iron uptake from soil [26]. Nevertheless, a potential interaction between PA biosynthesis and iron uptake efficiency is unknown. SAM is also substrate for ethylene biosynthesis in higher plants (Figure 2). The enzyme 1-aminocyclopropane-1-carboxylate (ACC) synthase converts SAM to ACC, which is oxidized by ACC oxidase to ethylene [27,28]. Hence, PAs and ethylene could act in an antagonistic manner competing for the common substrate SAM. Whereas ethylene would contribute to senescence and fruit ripening, PAs would favor growth and inhibit senescence [29]. Indeed, anti-senescent properties of PAs are well documented [30]. Some of the PA-related antisenescence effects might be due to the PA-mediated inhibition of ethylene biosynthesis. Indeed, examples are found in literature that the exogenous application of PAs inhibits ethylene 
biosynthesis [31-33]. In tomato, PAs have been shown to inhibit the induction of ACC synthase by wounding [34]. In carnation flowers [35] the blockage of SAM to ACC conversion was translated in an increase of Spm levels whereas impairment of PA biosynthesis promoted ethylene pathway and senescence. However, most of these analyses are based on exogenous applications of PAs, ethylene and their inhibitors which have some intrinsic limitations. Furthermore, the antagonistic effect between PAs and ethylene is not always observable. Mehta et al. [36] reported that tomato plants transformed with yeast $S A M D C$ under the control of the ripening specific E8 promoter synthesized more ethylene and PAs concomitantly during fruit ripening, thus evidencing the absence for SAM competition in this system. Initial studies already pointed to other mechanisms rather than a mere metabolic competition for SAM involved in the PA-ethylene antagonism [31-33] although scarce attention has been taken in the recent years to the PA-ethylene crosstalk. In a recent work, overexpression of yeast Spermidine Synthase in tomato has been shown to produce plants susceptible to the fungal necrotroph Botritis cynerea concomitantly to a repression of ethylene biosynthesis and signaling, thus providing support to the view that PA-ethylene cross-regulation occurs and has important implications for defense [37]. Further analyses would be required to identify the interacting nodes in this PA-ethylene cross-regulation.

\subsection{Put to Spm Canalization and Its Contribution to Stress Protection}

Higher PAs, Spd and Spm have been shown to significantly contribute to abiotic stress protection although their specific mechanism(s) of action remain to be elucidated. Some of the genetic evidences have been obtained from the analysis of loss-of-function mutations in Arabidopsis thaliana. This species carries two genes coding for Spd Synthase (SPDS1 and SPDS2), and one Spm Synthase (SPMS) [4]. Interestingly, Arabidopsis thaliana also contains one gene (ACAULIS5, ACL5) coding for thermospermine synthase, an enzyme involved in the synthesis of the Spm isomer thermospermine (tSpm) [38]. Regardless of its low levels, tSpm is required for proper vascular tissue development and stem elongation, phenotypes which are evidenced in the acl5 mutant [39]. The spms-1 mutant impared in Spm biosynthesis has no evident morphological alterations and it is viable [40]. The double spms-1/acl5-1 mutant lacking both Spm and tSpm is also viable and shows an acl5-1 phenotype $[39,40]$. However, impairment of Put or Spd biosynthesis leads to embryo lethality $[41,42]$. Consistent with a protective role of Spm and/or tSpm under abiotic stress, the double spms-1/acl5-1 mutant has also been reported to exhibit enhanced sensitivity to drought and salinity [43,44]. A potential role for tSpm in biotic stress protection in Arabidopsis thaliana has recently been proposed [45].

In a recent report [46], a Put to Spm metabolic canalization in response to drought was revealed in Arabidopsis thaliana and the resurrection plant Craterostigma plantagineum. In this work, the levels of PAs were analyzed in response to drought in Arabidopsis thaliana mutants impaired at different steps of the PA biosynthetic pathway. This approach allowed monitoring the accumulation of PAs during the course of dehydration. In this way, it could be revealed that even though Arabidopsis thaliana wild type plants do not accumulate Spd or Spm in response to drought, a Put to Spm canalization occurs which is not translated in Spd or Spm increases. Conversely, the drought tolerant species Craterostigma plantagineum showed dramatic increases in Spd and Spm contents that correlated with drought tolerance. Put to Spm metabolic canalization seems to be a conserved 
mechanism between species, whereas the ability to accumulate high Spd and Spm levels (e.g. by inhibition of its oxidation) may discern between drought tolerant and intolerant species.

\section{Polyamine Catabolism. Interactions with GABA}

PAs are oxidatively deaminated by the action of amine oxidases. Diamine oxidases (DAOs) are copper-containing amine oxidases $(\mathrm{CuAO})$ which oxidize the diamines Put and cadaverine at the primary amino groups. 4-aminobutanal is produced from the oxidation of Put by DAO activities, concomitantly with the release of ammonia and hydrogen peroxide (Figure 3). Other class of amine oxidases are flavin-containing polyamine oxidases (PAO) involved in the terminal catabolism of Spd and Spm producing 4-aminobutanal or N-(3-aminopropyl)-4-aminobutanal, 1,3-diaminopropane and $\mathrm{H}_{2} \mathrm{O}_{2}$ [47,48] (Figure 3). Another group of PAOs are involved in the back-conversion of Spm to Spd with concomitant production of 3-aminopropanal and $\mathrm{H}_{2} \mathrm{O}_{2}$ [48]. 4-aminobutanal produced in Put catabolism by DAO and terminal catabolism of Spd can be converted to $\gamma$-aminobutyric acid (GABA) via $\Delta 1$-pyrroline. It is well established the role of GABA as neurotransmitter in animal cells. In plants, increases in GABA levels have been reported in response to different stresses [49] and GABA is suggested to contribute to stress protection through the regulation of cellular $\mathrm{pH}$, acting as osmoregulator or as signaling molecule in plants [50]. However, most of the roles for GABA under stress still need to be defined. In soybean roots exposed to salinity, the degradation of PAs has been associated with increased levels of GABA [51]. Conversely, during the recovery from stress, the levels of GABA are reduced concomitantly to an increase of PAs. [51] These observations suggest that PA catabolism might contribute to increase GABA levels during salinity.

PA oxidation is a source of $\mathrm{H}_{2} \mathrm{O}_{2}$ in the apoplast which can contribute to the defense response against pathogens [47]. Evidences are found that some PAOs participate in the hypersensitive response (HR) in Nicotiana tabacum plants resistant to tobacco mosaic virus (TMV). Inhibition of PAO activity by guazatine reduced HR symptoms in TMV infected plants [52]. In a more recent work, $\mathrm{H}_{2} \mathrm{O}_{2}$ derived from PA catabolism has also been associated with non-host induced HR in tobacco, thus leading to the view that PAs could participate in both host and non-host induced HR [53]. 
Figure 3. Polyamine catabolism mediated by CuAOs (copper amine oxidases) and PAOs (polyamine oxidases) in plants.

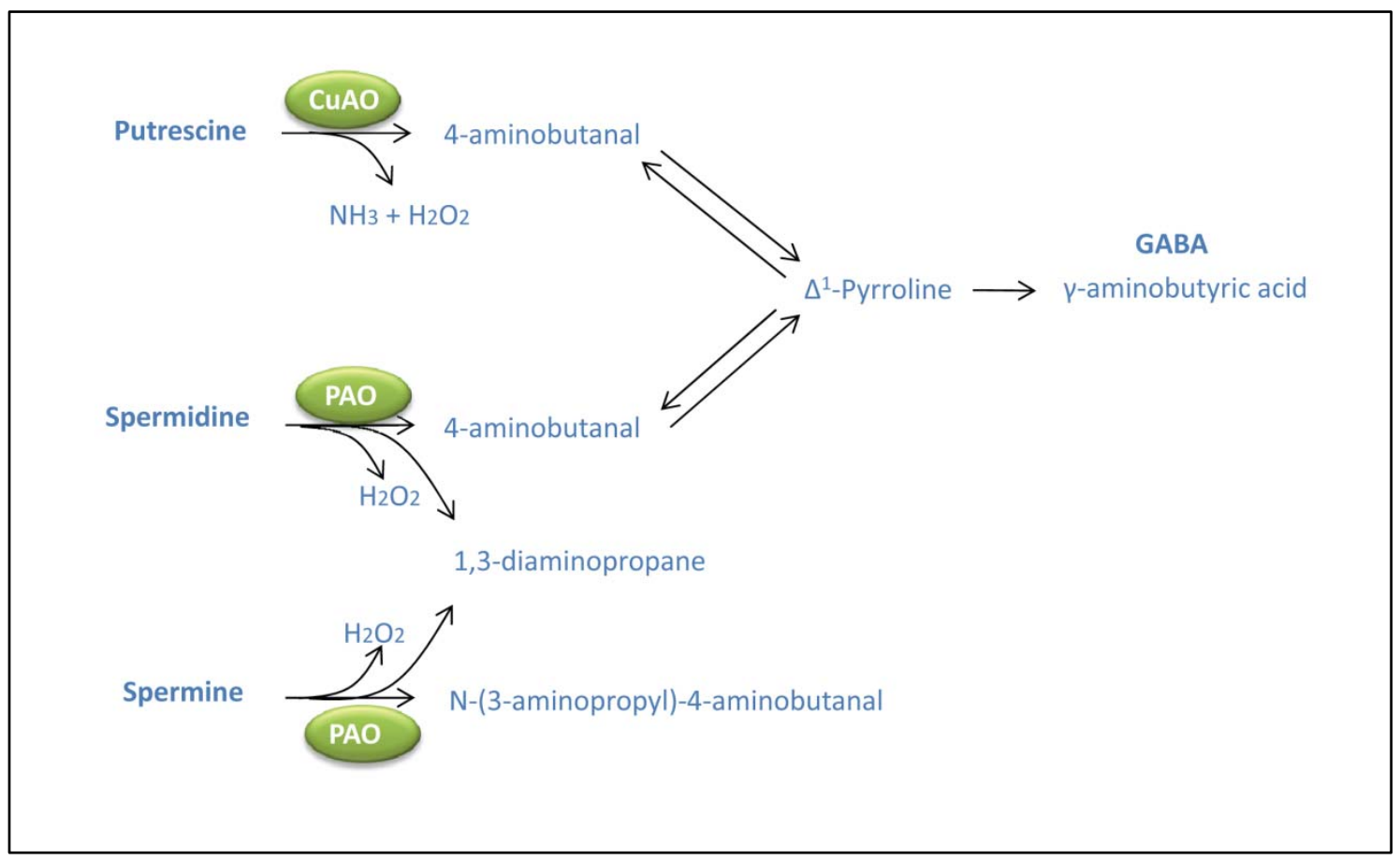

\section{ABA Dependence of PA-Responses under Drought}

Abscisic acid (ABA) is a key hormone with important roles in abiotic stress [54]. Many drought-inducible genes are responsive to ABA, but also ABA-independent pathways are activated in response to drought conditions. In order to determine the involvement of $\mathrm{ABA}$ in the transcriptional regulation of the PA biosynthetic pathway in response to drought, Alcázar et al. [55] analyzed the expression of PA biosynthesis genes ADC1, ADC2, AIH, CPA, SPDS1, SPDS2, SPMS, ACL5, $S A M D C 1$ and $S A M D C 2$ in Arabidopsis thaliana wild type plants and mutants impaired in ABA biosynthesis (aba2-3) or signaling (abi1-1). ADC2, SPDS1 and SPMS genes were among the most responsive ones to drought stress [55]. The increased expression of these three PA biosynthesis genes suggested a potential role for $A D C 2, S P D S 1$ and $S P M S$ in the drought response. Interestingly, whereas $A D C 2$ and $S P D S 1$ expression increased several fold by drought treatment, the expression of their gene paralogs, $A D C 1$ and $S P D S 2$, did not change substantially [55]. These observations are consistent with the acquisition of certain stress-specificity, probably due to divergent evolution of cis- regulatory elements in their promoters. Indeed, different cis elements are found in the promoters of PA biosynthesis genes [39,56]. ABA-responsive elements (ABRE) or ABRE-related motifs are also found in the promoters of $A D C 2, S P D S 1$ and SPMS [56], which are highly up-regulated in response to drought [55]. The analysis in aba2-3 and abil-1 mutants showed much more moderate increases in $A D C 2, S P D S 1$ and SPMS expression [55]. These results evidenced that transcriptional up-regulation of $A D C 2$, SDPS1 and SPMS by drought is mediated by ABA. Hence, ABA is an upstream regulator of PA biosynthesis in response to drought. To determine the effect of the transcriptional regulation of PA biosynthesis genes on PA levels, the content of Put, Spd and Spm levels in response to drought were also analyzed. Wild type plants showed a progressive accumulation of Put in response to drought 
conditions, whereas this accumulation was absent in aba2-3 and abil-1 [55]. Hence, the ABAdependent up-regulation in $A D C 2$ expression observed under drought leads to an effective Put accumulation. In a recent work, Alcázar et al. [10] determined a role for $A D C 2$ overexpression in conferring drought tolerance by transformation of $A$. thaliana plants with the homologous $A D C 2$ gene under the constitutive CaMV 35s promoter. The different lines analyzed showed contrasting degrees of $A D C 2$ expression and Put accumulation [9]. Total Put content was between 12- and 2-fold higher than wild type depending on the transgenic line $[9,10]$. Interestingly, plants that accumulated higher levels of Put were more resistant to drought stress and the enhanced drought tolerance correlated with a reduced stomata aperture and transpiration rate [10].

\section{Future Perspectives}

The polyamine field is complex. First because PAs have multiple roles acquired during evolution and it is difficult to disentangle one from the other to study them in isolation. Lethality due to depletion of PA levels is a good example of the intrinsic difficulties of addressing functional questions. To gain a further insight into this topic, we believe that it is necessary to study the interconnections of PA biosynthesis, degradation and conjugation with other metabolic routes in depth and place these compounds in the context of a full metabolic and signaling network. We still need to address where PAs localize, where they are required for their function, how they are transported from sources to sinks (and also which are the sources and the sinks) and more importantly, whether PAs are intermediary compounds in the stress protection or have a role themselves. We believe that a detailed metabolic and signaling analysis addressing these and other fundamental questions is needed to provide a broader view about the roles and mechanisms of PAs during stress.

\section{Acknowledgments}

We apologize to authors whose work has not been cited here owing to space limitations. R.A. acknowledges support from the Ramón y Cajal Programme of the Ministry of Economy and Competitiveness, Spain. Research in our laboratory is financed by Spanish Ministry of Science and Education (BIO2008-05493-C02-01 and BIO2011-2963) and by the Generalitat de Catalunya (2009SGR1060).

\section{Conflict of Interest}

The authors declare no conflict of interest.

\section{References}

1. Porter, J.R. Rising temperatures are likely to reduce crop yields. Nature 2005, 436, 17 4-174.

2. Thuiller, W.; Lavorel, S.; Araujo, M.B.; Sykes, M.T.; Prentice, I.C. Climate change threats to plant diversity in Europe. Proc. Natl. Acad. Sci. USA 2005, 102, 8245-8250.

3. Bhatnagar-Mathur, P.; Vadez, V.; Sharma, K.K. Transgenic approaches for abiotic stress tolerance in plants: retrospect and prospects. Plant Cell Rep. 2008, 27, 411-424. 
4. Alcázar, R.; Altabella, T.; Marco, F.; Bortolotti, C.; Reymond, M.; Koncz, C.; Carrasco, P.; Tiburcio, A.F. Polyamines: Molecules with regulatory functions in plant abiotic stress tolerance. Planta 2010, 231, 1237-1249.

5. Martin-Tanguy, J. Conjugated polyamines and reproductive development: Biochemical, molecular and physiological approaches. Physiol. Plant. 1997, 100, 675-688.

6. Hanfrey, C.; Sommer, S.; Mayer, M.J.; Burtin, D.; Michael, A.J. Arabidopsis polyamine biosynthesis: absence of ornithine decarboxylase and the mechanism of arginine decarboxylase activity. Plant J. 2001, 27, 551-560.

7. Murakami, Y.; Matsufuji, S.; Kameji, T.; Hayashi, S.; Igarashi, K.; Tamura, T.; Tanaka, K.; Ichihara, A. Ornithine decarboxylase is degraded by the 26s-proteasome without ubiquitination. Nature 1992, 360, 597-599.

8. Kahana, C. Ubiquitin dependent and independent protein degradation in the regulation of cellular polyamines. Amino Acids 2007, 33, 225-230.

9. Alcázar, R.; García-Martínez, J.L.; Cuevas, J.C.; Tiburcio, A.F.; Altabella, T. Overexpression of $A D C 2$ in Arabidopsis induces dwarfism and late-flowering through GA deficiency. Plant J. 2005, 43, 425-436.

10. Alcázar, R.; Planas, J.; Saxena, T.; Zarza, X.; Bortolotti, C.; Cuevas, J.; Bitrián, M.; Tiburcio, A.; Altabella, T. Putrescine accumulation confers drought tolerance in transgenic Arabidopsis plants over-expressing the homologous Arginine decarboxylase 2 gene. Plant Physiol. Biochem. 2010, 48, 547-522.

11. Krumpelman, P.M.; Freyermuth, S.K.; Cannon, J.F.; Fink, G.R.; Polacco, J.C. Nucleotide sequence of Arabidopsis thaliana arginase expressed in yeast. Plant Physiol. 1995, 107, 1479-1480.

12. Brownfield, D.L.; Todd, C.D.; Deyholos, M.K. Analysis of Arabidopsis arginase gene transcription patterns indicates specific biological functions for recently diverged paralogs. Plant Mol. Biol. 2008, 67, 429-440.

13. Flores, T.; Todd, C.D.; Tovar-Mendez, A.; Dhanoa, P.K.; Correa-Aragunde, N.; Hoyos, M.E.; Brownfield, D.M.; Mullen, R.T.; Lamattina, L.; Polacco, J.C. Arginase-negative mutants of Arabidopsis exhibit increased nitric oxide signaling in root development. Plant Physiol. 2008, 147, 1936-1946.

14. Brauc, S.; De Vooght, E.; Claeys, M.; Geuns, J.M.C.; Hofte, M.; Angenon, G. Overexpression of arginase in Arabidopsis thaliana influences defence responses against Botrytis cinerea. Plant Biol. 2012, 14, 39-45.

15. Witte, C.-P. Urea metabolism in plants. Plant Sci. 2011, 180, 431-438.

16. Funck, D.; Stadelhofer, B.; Koch, W. Ornithine-delta-aminotransferase is essential for arginine catabolism but not for proline biosynthesis. BMC Plant Biol. 2008, 8 .

17. Hancock, J.T.; Neill, S.J.; Wilson, I.D. Nitric oxide and ABA in the control of plant function. Plant Sci. 2011, 181, 555-559.

18. Crawford, N.M.; Galli, M.; Tischner, R.; Heimer, Y.M.; Okamoto, M.; Mack, A. Plant nitric oxide synthase: back to square one-Response. Trends Plant Sci. 2006, 11, 526-527. 
19. Tun, N.N.; Santa-Catarina, C.; Begum, T.; Silveira, V.; Handro, W.; Floh, E.I.S.; Scherer, G.F.E. Polyamines induce rapid biosynthesis of nitric oxide (NO) in Arabidopsis thaliana seedlings. Plant Cell Physiol. 2006, 47, 346-354.

20. Yamasaki, H.; Cohen, M.F. NO signal at the crossroads: polyamine-induced nitric oxide synthesis in plants? Trends Plant Sci. 2006, 11, 522-524.

21. Biastoff, S.; Brandt, W.; Draeger, B. Putrescine N-methyltransferase - The start for alkaloids. Phytochemistry 2009, 70, 1708-1718.

22. Ge, C.M.; Cui, X.; Wang, Y.H.; Hu, Y.X.; Fu, Z.M.; Zhang, D.F.; Cheng, Z.K.; Li, J.Y. BUD2, encoding an S-adenosylmethionine decarboxylase, is required for Arabidopsis growth and development. Cell Res. 2006, 16, 446-456.

23. Roje, S. S-Adenosyl-L-methionine: Beyond the universal methyl group donor. Phytochemistry 2006, 67, 1686-1698.

24. Pegg, A.E. S-Adenosylmethionine decarboxylase. In Essays in Biochemistry, Vol 46: The Polyamines: Small Molecules in the Omics Era; Wallace, H., Ed.; Portland Press Ltd: London, UK, 2009; Volume 46, pp. 25-45.

25. Herbik, A.; Koch, G.; Mock, H.P.; Dushkov, D.; Czihal, A.; Thielmann, J.; Stephan, U.W.; Baumlein, H. Isolation, characterization and cDNA cloning of nicotianamine synthase from barley - A key enzyme for iron homeostasis in plants. Eur. J. Biochem. 1999, 265, 231-239.

26. Mizuno, D.; Higuchi, K.; Sakamoto, T.; Nakanishi, H.; Mori, S.; Nishizawa, N.K. Three nicotianamine synthase genes isolated from maize are differentially regulated by iron nutritional status. Plant Physiol. 2003, 132, 1989-1997.

27. Fluhr, R.; Mattoo, A.K. Ethylene - Biosynthesis and perception. Crc. Cr. Rev. Plant Sci. 1996, 15, 479-523.

28. Yang, S.F.; Hoffman, N.E. Ethylene biosynthesis and its regulation in higher-plants. Ann. Rev. Plant Phys. 1984, 35, 155-189.

29. Mattoo, A.K.; Suttle, J.C. The Plant Hormone Ethylene; CRC Press: Boca Raton: FL, USA, 1991; 21-42.

30. Evans, P.T.; Malmberg, R.L. Do polyamines have roles in plant development. Ann. Rev. Plant Phys. 1989, 40, 235-269.

31. Apelbaum, A.; Burgoon, A.C.; Anderson, J.D.; Lieberman, M.; Benarie, R.; Mattoo, A.K. Polyamines inhibit biosynthesis of ethylene in higher-plant tissue and fruit protoplasts. Plant Physiol. 1981, 68, 453-456.

32. Apelbaum, A.; Goldlust, A.; Icekson, I. Control by ethylene of arginine decarboxylase activity in pea-seedlings and its implication for hormonal-regulation of plant-growth. Plant Physiol. 1985, 79, 635-640.

33. Suttle, J.C. Effect of polyamines on ethylene production. Phytochemistry 1981, 20, 1477-1480.

34. Li, N.; Parsons, B.L.; Liu, D.R.; Mattoo, A.K. Accumulation of wound-inducible acc synthase transcript in tomato fruit is inhibited by salicylic-acid and polyamines. Plant Mol. Biol. 1992, 18, 477-487.

35. Roberts, D.R.; Walker, M.A.; Thompson, J.E.; Dumbroff, E.B. The effects of inhibitors of polyamine and ethylene biosynthesis on senescence, ethylene production and polyamine levels in cut carnation flowers. Plant Cell Physiol. 1984, 25, 315-322. 
36. Mehta, R.A.; Cassol, T.; Li, N.; Ali, N.; Handa, A.K.; Mattoo, A.K. Engineered polyamine accumulation in tomato enhances phytonutrient content, juice quality, and vine life. Nat. Biotechnol. 2002, 20, 613-618.

37. Nambeesan, S.; AbuQamar, S.; Laluk, K.; Mattoo, A.K.; Mickelbart, M.V.; Ferruzzi, M.G.; Mengiste, T.; Handa, A.K. Polyamines attenuate ethylene-mediated defense responses to abrogate resistance to Botrytis cinerea in tomato. Plant Physiol. 2012, 158, 1034-1045.

38. Knott, J.M.; Romer, P.; Sumper, M. Putative spermine synthases from Thalassiosira pseudonana and Arabidopsis thaliana synthesize thermospermine rather than spermine. FEBS Lett. 2007, 581, 3081-3086.

39. Hanzawa, Y.; Takahashi, T.; Michael, A.J.; Burtin, D.; Long, D.; Pineiro, M.; Coupland, G.; Komeda, Y. ACAULIS5, an Arabidopsis gene required for stem elongation, encodes a spermine synthase. EMBO J. 2000, 19, 4248-4256.

40. Imai, A.; Akiyama, T.; Kato, T.; Sato, S.; Tabata, S.; Yamamoto, K.T.; Takahashi, T. Spermine is not essential for survival of Arabidopsis. FEBS Lett. 2004, 556, 148-152.

41. Imai, A.; Matsuyama, T.; Hanzawa, Y.; Akiyama, T.; Tamaoki, M.; Saji, H.; Shirano, Y.; Kato, T.; Hayashi, H.; Shibata, D.; Tabata, S.; Komeda, Y.; Takahashi, T. Spermidine synthase genes are essential for survival of Arabidopsis. Plant Physiol. 2004, 135, 1565-1573.

42. Urano, K.; Hobo, T.; Shinozaki, K. Arabidopsis ADC genes involved in polyamine biosynthesis are essential for seed development. FEBS Lett. 2005, 579, 1557-1564.

43. Yamaguchi, K.; Takahashi, Y.; Berberich, T.; Imai, A.; Miyazaki, A.; Takahashi, T.; Michael, A.; Kusano, T. The polyamine spermine protects against high salt stress in Arabidopsis thaliana. FEBS Lett. 2006, 580, 6783-6788.

44. Yamaguchi, K.; Takahashi, Y.; Berberich, T.; Imai, A.; Takahashi, T.; Michael, A.J.; Kusano, T. A protective role for the polyamine spermine against drought stress in Arabidopsis. Biochem. Biophys. Res. Commun. 2007, 352, 486-490.

45. Sagor, G.H.M.; Takahashi, H.; Niitsu, M.; Takahashi, Y.; Berberich, T.; Kusano, T. Exogenous thermospermine has an activity to induce a subset of the defense genes and restrict cucumber mosaic virus multiplication in Arabidopsis thaliana. Plant Cell Rep. 2012, 31, 1227-1232.

46. Alcázar, R.; Bitrián, M.; Bartels, D.; Koncz, C.; Altabella, T.; Tiburcio, A.F. Polyamine metabolic canalization in response to drought stress in Arabidopsis and the resurrection plant Craterostigma plantagineum. Plant Signal. Behav. 2011, 6, 243-250.

47. Cona, A.; Rea, G.; Angelini, R.; Federico, R.; Tavladoraki, P. Functions of amine oxidases in plant development and defence. Trends Plant Sci. 2006, 11, 80-88.

48. Moschou, P.N.; Paschalidis, K.A.; Roubelakis-Angelakis, K.A. Plant polyamine catabolism: The state of the art. Plant Signal. Behav. 2008, 3, 1061-1066.

49. Roberts, M.R. Does GABA act as a signal in plants? Hints from molecular studies. Plant Signal. Behav. 2007, 2, 408-409.

50. Bouché, N.; Fromm, H. GABA in plants: just a metabolite? Trends Plant Sci. 2004, 9, 110-115.

51. Xing, S.G.; Jun, Y.B.; Hau, Z.W.; Liang, L.Y. Higher accumulation of $\gamma$-aminobutyric acid induced by salt stress through stimulating the activity of diamine oxidases in Glycine max (L.) Merr. roots. Plant Physiol. Biochem. 2007, 45, 560-566. 
52. Yoda, H.; Yamaguchi, Y.; Sano, H. Induction of hypersensitive cell death by hydrogen peroxide produced through polyamine degradation in tobacco plants. Plant Physiol. 2003, 132, 1973-1981.

53. Yoda, H.; Fujimura, K.; Takahashi, H.; Munemura, I.; Uchimiya, H.; Sano, H. Polyamines as a common source of hydrogen peroxide in host- and nonhost hypersensitive response during pathogen infection. Plant Mol. Biol. 2009, 70, 103-112.

54. Yamaguchi-Shinozaki, K.; Shinozaki, K. Transcriptional regulatory networks in cellular responses and tolerance to dehydration and cold stresses. Annu. Rev. Plant Biol. 2006, 57, 781-803.

55. Alcázar, R.; Cuevas, J.C.; Patrón, M.; Altabella, T.; Tiburcio, A.F. Abscisic acid modulates polyamine metabolism under water stress in Arabidopsis thaliana. Physiol. Plant. 2006, 128, 448-455.

56. Alcázar, R.; Marco, F.; Cuevas, J.C.; Patrón, M.; Ferrando, A.; Carrasco, P.; Tiburcio, A.F.; Altabella, T. Involvement of polyamines in plant response to abiotic stress. Biotechnol Lett. 2006, $28,1867-1876$.

(C) 2012 by the authors; licensee MDPI, Basel, Switzerland. This article is an open access article distributed under the terms and conditions of the Creative Commons Attribution license (http://creativecommons.org/licenses/by/3.0/). 